\title{
Rapid Osteolysis and Loosening of Unicompartmantal Knee Prosthesis Due to Posterior Dislocation of the Polyethylene Insert
}

\author{
Alauddin Kochai* \\ Department of Orthopedic and Traumatology, Turkey \\ *Corresponding author: Alauddin Kochai, Department of Orthopedic and Traumatology, Turkey \\ Submission: 眥 March 30, 2018; Published: 眥 November 13, 2018
}

\begin{abstract}
Introduction: Unicondylar knee arthroplasty (UKA) is a treatment option for pure medial tibiofemoral osteoarthrosis. The most common complication of UKA is anterior or medial polyethylene dislocation. We report a rare complication (posterior fixed polyethylene dislocation) of UKA.

Case: We present a case of 79-year-old man with bilateral medial compart man arthrosis underwent bilaterally medial UKA. He had posterior fixed intra-articular polyethylene dislocation which was missed by his first intervention. After three months of missed polyethylene dislocation the implants of UKA was totally loosen.

Conclusion: Diagnostic delay of polyethylene dislocation may result to loosening of component and it will lead to revision of UKA to TKA, which is technically challenging then primer total knee arthroplasty. The most common early complication of UKA is polyethylene dislocation. This should not be forgotten in a painful UKA with locking or without locking.
\end{abstract}

\section{Introduction}

Unicompartmantal knee arthroplasty (UKA) is a treatment option for pure medial tibiofemoral osteoarthrosis. Since being reported by Marmor [1] the frequency of UKA is gradually increasing with the advancement of minimal invasive technique and surgical instrument [1]. Also, less postoperative pain, less blood loss, earlier rehabilitation and better range of motion makes it popular option $[2,3]$. In addition, UKA is limited to a single compartment, it has the advantages of minimal bone resection, and preserving more normal knee functions TKA (Total Knee Arthroplasty) [4]. Despite these advantages, UKA also has disadvantages and complications such as surgical technique difficulties, infection, subluxation of the knee joint due to inaccuracy of implant implantation, bone defects developed during revision, early lateral compartment arthrosis, Proximal tibia fracture and polyethylene bearing dislocation $[5,6]$. The dislocation of polyethylene is usually anterior, and it is rare in other directions $[7,8]$. This study presents a case of posterior dislocation of polyethylene bearing which is a rare complication for UKA.

\section{Case Report}

A 79 years old man with bilateral medial compartment arthrosis underwent bilaterally UKA in a state hospital. The postoperative period was ordinary. Bilateral knee range of motion (ROM) was 0 to 110 degrees and didn't have any pain until 10 months after operation. The pain began suddenly after a long sitting period of knee 90-degree flexion. Without history of trauma. The patient had a permanent knee pain and diffuse swelling. Upon his visit to his surgeon, he was told that 'everything is ok' and the medical prescription was given. At his physical examination, the ROM was 0 to 110 degrees. It was like early postoperative period, but he had pain specially at hyperflexion in popliteal fossa. Anterior-posterior (AP) radiography was taken, but dislocation of the polyethylene was missed at those radiographies (Figure 1). His pain persisted and got worsen. The patient could walk hardly with a walking device. Fortyfive days after the symptoms began the patient visited our clinic. AP and lateral graphy of both knees taken, the polyethylene was dislocated and located at posterior of the knee in the right side. The patient had serious lung problems that should be treated first.

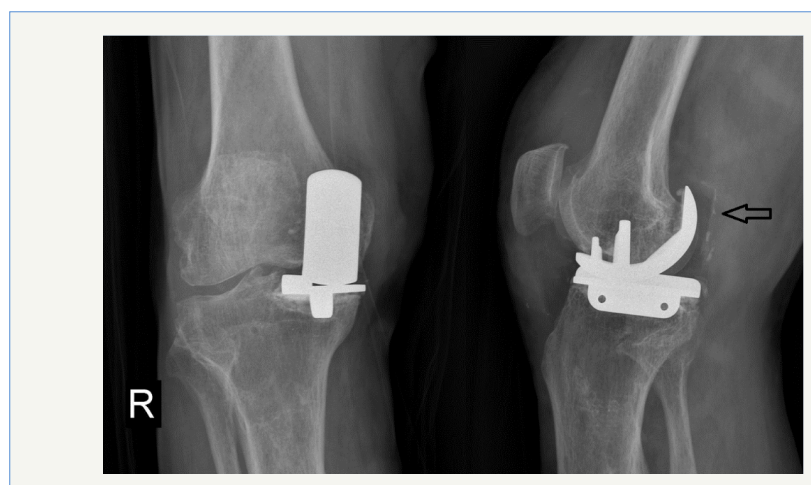

Figure 1 
The patient was referred to department of chest diseases and department of anesthesiology for preoperative preparation and treatment of lung problems. The department of chest diseases didn't allow for an elective operation. He had to continue medical treatment for his lung diseases. After one and a half month the department of chest diseases and anesthesiology allowed for operation. In AP graphy the femoral and tibial components were loosen (Figure 2). Revision of UKA to TKA planed. Previous incision and medial parapatellar approach for arthrotomy performed. All compartments of the knee were covered by metallizes. The tibial and femoral components were loose and were moving with flexion and extension of the knee. The polyethylene was fixed at the posterior of the knee. The tibia and femoral components easily removed. Polyethylene released from the posterior of the knee and removed. Femoral and tibia osteotomies for total knee arthroplasty performed. Total knee arthroplasty procedure successfully performed (Figure 3). At the first day after operation the patient began walking comfortably without any support. The patient was extern 4 days after operation with 0-120 degrees ROM of the knee.

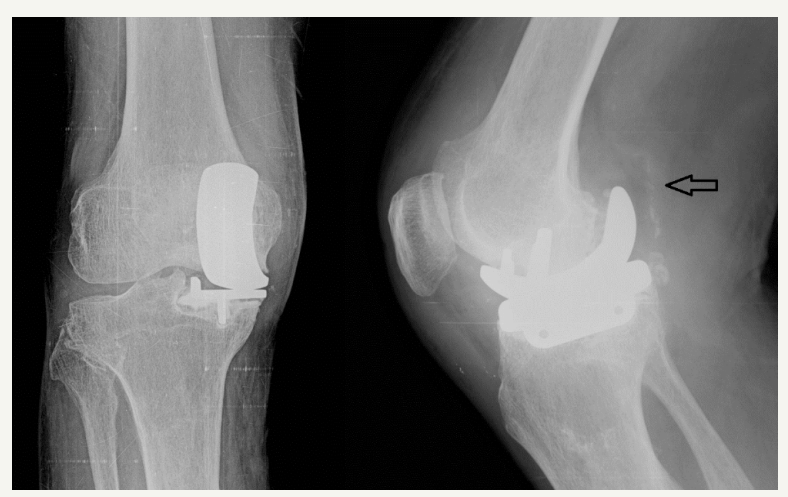

Figure 2

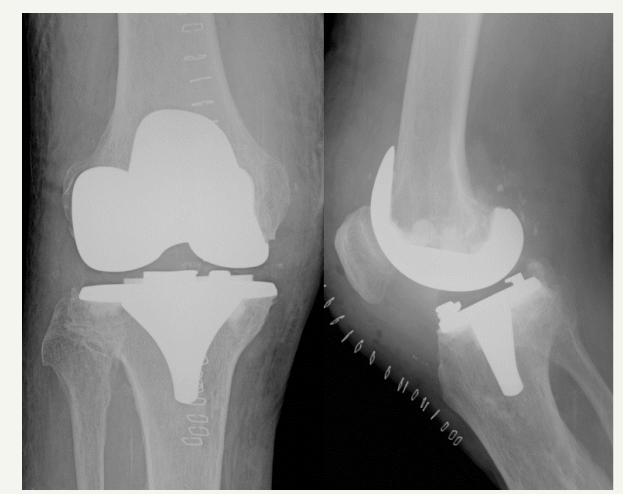

Figure 3

\section{Discussion}

Dislocation of polyethylene is the most common complication for mobile-bearing noncompartmental knee arthroplasty. The dislocation is usually anterior, lateral and medial side of the knee. Posterior dislocation is rare [7,9]. The previously reported posterior dislocations were extra-articular and were not fixed to the posterior of femoral condyle. In those cases, the posteriorly dislocated polyethylene was removed by a posterior incision $[10,11]$. In our case we removed the polyethylene with the anterior incision. The main clinical presentation of polyethylene dislocation is pain, mechanical locking of the knee and may be palpable if it is dislocation to anterior of medial. In this case polyethylene was dislocated intra-articular posteriorly, which was fixed to femoral condyle, so the patient didn't feel locking on his knee. This caused miss diagnosis at the first investigation of the patient.

Dislocation of polyethylene is mostly caused by a technical problem. It typically causes by posterior impingement of femoral osteophytes or remainder of the degenerative meniscus, maltracking of femoral component relative to the tibial component or ligamentous laxity [12,13]. In present case, the femoral component was implanted in flexion position, it was impinging to polyethylene. Which may be the main reason of dislocation in our case.

The loosening of implant may depend on malalignment of knee [14], tibial subsidence [15], ligament instability [16] and recurrence or missed polyethylene dislocation [17]. Missed or recurrence dislocations results to malalignment and changes the space of joint and mechanical forces to medial compartment. Changing of mechanical forces rules on aseptic loosening of UKA. In a study of Chatellard et al. [18] they provide that joint space changes in $61.8 \%$ of UKA. In $48.1 \%$ of these cases have lower joint space than the lateral side. Being lower at prosthetic side results to aseptic loosening. Being lower of lateral side results progression of lateral compartment osteoarthrosis [18]. In our case there wasn't any radiography sign of loosening at initially. The implant loosening seen in three months. Which can be explain by the changing of mechanical forces of medial side.

There are a lot of options for revision of failed UKA. The most common revisions are changing of polyethylene to thicker one, or revision of UKA to TKA [12,19]. Chou et al. [19] demonstrate that the functional results of revision TKA for failed UKR are inferior to those obtained after primary TKA. Also, revision surgery for failed UKA to TKA can be technically challenging to those of primary total knee arthroplasty [19].

\section{Conclusion}

Diagnostic delay of polyethylene dislocation may result to loosening of component and it will lead to revision of UKA to TKA, which is technically challenging then primer total knee arthroplasty. The most common early complication of UKA is polyethylene dislocation. This most not be forgotten in a painful UKA with locking or without locking.

\section{References}

1. Marmor L (1979) Marmor moduler knee in unicompartmantal diseases Minimum four-year follow-up. J Bone Joint Surg Am 61(3): 347-353.

2. Berger RA, Nedeff DD, Barden RM, Sheinkop MM, Jacobs JJ, et al. (1999) Unicompartmantal knee arthroplasty: Clinical exoerience at 6- to 10year followup. Clin Orthop Relat Res 367: 50-60. 
3. Riddle DL, Jiranek WA, Mcglynn FJ (2008) Yearly incidence of unicompartmantal knee arthroplasty in United State. J Arthroplasty 23(3): 408-412.

4. Chassin EP, Mikosz RP, Andriacchi TP, Rosenberg AG (1996) Functional analysis of cemented medial unicompartmental knee arthroplasty. J Arthroplasty 11(5): 553-559.

5. Aleto TJ, Berend ME, Ritter MA, Faris PM, Meneghini RM (2008) Early failure of unicompartmental knee arthroplasty leading to revision. J Arthroplasty. 23(2): 159-163.

6. Surendran S, Kwak DS, Lee UY, Park SE, Gopinathan P, et al. (2007) Anthropometry of the medial tibial condyle to design the tibial component for unicondylar knee arthroplasty for the Korean population. Knee Surg Sports Traumatol Arthrosc. 15(4): 436-442.

7. Robinson BJ, Rees JL, Price AJ, Beard DJ, Murray DW, et al. (2002) Dislocation of the bearing of the Oxford lateral unicompartmental arthroplasty. A radiological assessment. J Bone Joint Surg Br 84(5): 653657.

8. Verhaven E, Handelberg F, Casteleyn, Opdecam P (1991) Meniscal bearing dislocation in the Oxford knee. Acta Orthop Belg 57(4): 430-432.

9. Clark M, Campbell DG, Kiss G, Dobson PJ, Lewis PL (2010) Reintervention after mobile-bearing Oxford unicompartmental knee arthroplasty. Clin Orthop Relat Res 468(2): 576-580.

10. Jeong JH, Kang H, Ha YC, Eui Chan Jang (2012) Incarceration of a dislocated mobile bearing to the popliteal fossa after unicompartmental knee arthroplasty. The Journal of Arthroplasty 27(2): 323.e5-323.e7.

11. Singh VK, Apsingi S, Balakrishnan S, Manjure S (2012) Posterior dislocation of meniscal bearing insert in mobile bearing unicompartmental knee. Arthroplasty mimicking a baker cyst. The Journal of Arthroplasty 27(3): 494.e13-494.e16.
12. Song MH, Kim BH, Ahn SJ, Seong Ho Y, Min Soo L (2009) Early complications after minimally invasive mobile-bearing medial unicompartmental knee arthroplasty. J Arthroplasty 24(8): 1281-1284.

13. Vardi G, Strover AE (2004) Early complications of unicompartmental knee replacement: The Droitwich experience. Knee 11(5): 389-394.

14. Bert JM, Smith R (1997) Failures of metal-backed unicompartmental arthroplasty. Knee 4(1): 41-48.

15. Zermatten P, Munzinger U (2012) The Oxford II medial unicompartmental knee arthroplasty: an independent 10-year survival study. Acta Orthop Belg 78(2): 203-209.

16. Citak M, Dersch K, Kamath AF, Haasper C, Gehrke T, et al. (2014) Common causes of failed unicompartmentalknee arthroplasty: a singlecentre analysis of four hundred and seventy-one cases. Int Orthop 38(5): 961-965.

17. Wynn JH, Chan W, Harrison T, Smith TO, Masonda P, et al. (2012) Revision of medial Oxford unicompartmental knee replacement to a total knee replacement: similar to a primary? Knee 19(4): 339-343.

18. Chatellard R, Sauleau V, Colmar M, Robert H, Raynaud G, et al. (2013) Medial unicompartmental knee arthroplasty: does tibial component position influence clinical outcomes and arthroplasty survival? Orthop Traumatol Surg Res 99(4): 219-225.

19. Chou DT, Swamy GN, Lewis JR, Badhe NP (2012) Revision of failed unicompartmental knee replacement to total knee replacement. Knee 19(4): 356-359.
Creative Commons Attribution 4.0

International License

For possible submissions Click Here

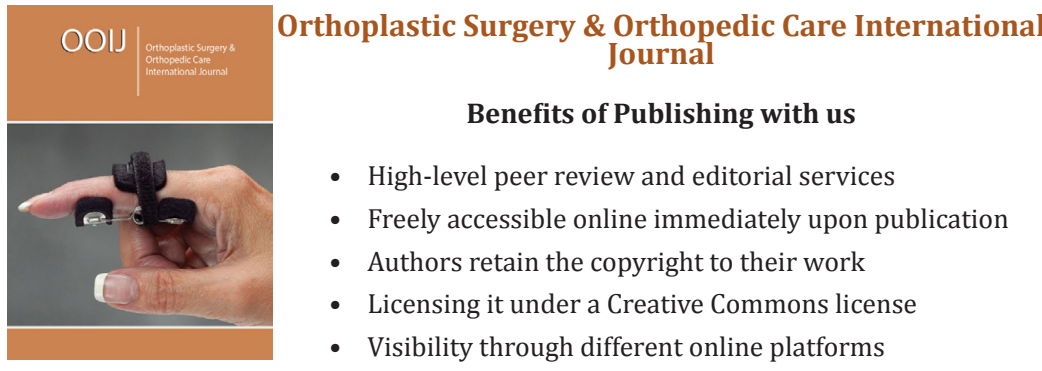

